\title{
An Efficient Damage Identification Method for Simply Supported Beams Based on Strain Energy Information Entropy
}

\author{
Chang-Sheng Xiang, ${ }^{1}$ Ling-Yun Li, ${ }^{1}$ Yu Zhou $\mathbb{D}^{2},{ }^{2}$ and Cong Dang $\mathbb{D}^{3}$ \\ ${ }^{1}$ School of Civil Engineering, Lanzhou University of Technology, Lanzhou 730050, China \\ ${ }^{2}$ College of Civil Engineering, Anhui Jianzhu University, Hefei 230601, China \\ ${ }^{3}$ Zhejiang Zhongnan Curtain Wall Co, Ltd, Hangzhou 310052, China \\ Correspondence should be addressed to Yu Zhou; yuzhou923@outlook.com
}

Received 23 April 2020; Revised 12 August 2020; Accepted 17 August 2020; Published 7 September 2020

Academic Editor: Hamed Akhavan

Copyright ( 92020 Chang-Sheng Xiang et al. This is an open access article distributed under the Creative Commons Attribution License, which permits unrestricted use, distribution, and reproduction in any medium, provided the original work is properly cited.

\begin{abstract}
Concrete simply supported beams are widely used in small- and medium-sized bridges. Hinged slabs and joints are damaged in service easily, as they are the main load-carrying members of bridge. In this paper, using the high sensitivity and strong antinoise characteristics of strain energy to damage, a strain energy information entropy index is proposed for identifying local damage in hinge joints and hinged slabs. Moreover, a new damage index of the local strain energy entropy function is constructed according to the strain energy of a hinge joint element and its surrounding elements. A simply supported beam model is established to verify the effectiveness of the proposed index. The numerical simulation results show that the proposed damage index of hinge joints can accurately locate and quantify the degree of damage of single-hinge joints and multihinge joints. The improved modal strain energy information entropy index is sensitive to single-hinge slab damage and can accurately identify damage with noise levels of $5 \%$ and $10 \%$.
\end{abstract}

\section{Introduction}

The simply supported beam is widely used in small- and medium-sized span bridges due to its simple structure, convenient construction, and mature technology. It consists of a number of concrete slabs connected by tongue-andgroove concrete joints to bear vehicle loads [1-3]. With time, the bridge inevitably suffers structural damage under the passing of heavy loads. Most of the damage occurs in the essential parts of the bridge; specifically, the joints between hinged slabs are easily damaged [4-6]. In one area of China, 402 out of 429 simply supported beam bridges have cracks at the bottom of hinged slabs, with an incidence of about $93.5 \%$, as reported in the survey data of Qin et al. [7]. Moreover, 412 bridges had hinge joints damaged by water seepage and concrete debris, with an incidence of about $96.0 \%$. Therefore, the working state of simply supported beam bridges should be considered and evaluated for further operation and maintenance.
With the development of technology, a number of structural damage detection techniques have been developed in the past few decades. One of these, the vibration-based techniques, have drawn much attention by researchers, such as the methods based on modal frequency [8], modal shape or mode shape derivatives [9-11], modal flexibility [12], modal strain energy (MSE) [13], and so on. Modal strain energy performs particularly well, showing a good sensitivity to damage of structural. Shi et al. [13] were the first to use the MSE method to identify structural damage; only a few lower displacement modes were used to calculate the MSE before and after damage. Shi et al. [14] also proposed an improved MSE method that significantly reduces the modal truncation error and finite-element modeling errors from the analytical model in the computations. A two-stage structural damage detection method based on MSE and particle swarm optimization was proposed by Seyedpoor [15] to improve the accuracy of damage detection. Jahangiri [16] proposed a novel identification method for structural damage 
assessment using the first vibration mode data, which not only predicts the damage location properly but also estimates the damage intensity successfully. The MSE method usually requires complete information of structural mode shapes. However, complete modal shape information is hard to obtain due to the limited number of sensors and the difficulty of measuring rotational vibration. Wu [17] proposed a novel approach, where the displacement mode and rotational mode included in the definition of the elemental MSE are replaced by strain mode, effectively improving the accuracy of the MSE.

The randomness and uncertainty of actual structural damage generally bring great challenge to the damage condition assessment. Although the traditional damage identification method based on structural vibration characteristics performs well in damage location, there are still many defects in damage quantitative analysis. Therefore, the information entropy theory, which describes the uncertainty of the system, is combined with the characteristic parameters of structural vibration to measure the damage of the structure from the perspective of probability. Recently, the information entropy theory has been introduced into structural damage identification and has been applied in dam safety analysis [18], surrounding rock stability [19], mechanical bearing damage [20], and other fields. However, there are few published studies on the field of structural beam damage. Liu et al. $[21,22]$ introduced the information entropy theory into the dynamic damage identification of concrete structures, providing a new idea for solving problems such as insufficient sensitivity and difficulty in nonlinear analysis for damage identification. Moreover, some scholars have successfully applied the information entropy theory to the research on crack propagation mechanism of concrete structures and achieved good results $[23,24]$.

In this paper, by combining the strain energy theory with the theory of information entropy, an index of information entropy of strain energy is proposed and studied to identify damage at hinge joints and hinged slabs.

\section{Theoretical Basis of Damage Identification Method}

2.1. Information Entropy Theory. Entropy is a special parameter in thermodynamics and statistical physics; it was proposed by the famous German physicist Clausius in 1865 . Entropy is a state variable, and the change in entropy relates to the dispersion of matter; furthermore, its macroscopic definition indicates the degree of uniformity of the energy distribution in the system. In 1948, C. E. Shannon proposed the concept of information entropy together with the theory of probability and statistics, which has been used as a parameter to measure the complexity of time series, and it can also be used to solve the problem of quantitative measurement [25].

If the variable $X$ has $n$ possible states $x_{i}, i=1,2, \ldots, n$, the probability $p\left(x_{i}\right), i=1,2, \ldots, n$, meets the following requirements:

$$
\begin{aligned}
& 0 \leq p\left(x_{i}\right) \leq 1 . \\
& \sum_{i=1}^{n} p\left(x_{i}\right)=1 .
\end{aligned}
$$

The amount of information represented by each state $x_{i}$ is $I\left(x_{i}\right)=-\ln p\left(x_{i}\right)$, where $i=1,2, \ldots, n$, which is known as the self-information of variable $X$. If there is high uncertainty in a specific state of $X$, then the probability of this state pattern will be small, and vice versa [26]. The information entropy can be defined as the expected value of the variable, as follows:

$$
H(X)=E\left[I\left(x_{i}\right)\right]=-\sum_{i=1}^{n} p\left(x_{i}\right) \ln p\left(x_{i}\right) .
$$

It can also be regarded as a probability distribution function of variable $X$. If the variable is orderly, the value of entropy is small. Conversely, if the variable is irregular, the value of entropy is high. Thus, the entropy function can be regarded as a parameter to measure the complexity of the time series, and it can also be used to solve the problem of quantitative measurement.

2.2. Strain Energy Calculation. The elastic strain energy of the concrete hinged slab element $i$ is known from the theoretical basis of finite elements:

$$
W_{s i}=\left(\frac{1}{2}\right) \sum_{k=1}^{n_{\text {int }}}\left\{\sigma^{e}\right\}^{T}\left\{\varepsilon^{e}\right\} \operatorname{vol}_{k},
$$

where $W_{s i}$ is the strain energy of the concrete hinged slab element $i ; n_{\text {int }}$ is the number of unit integration points; $\left\{\sigma^{e}\right\}$ is the stress vector; $\left\{\varepsilon^{e}\right\}$ is the elastic strain vector; and $\operatorname{vol}_{k}$ is the volume of unit integration point $k$.

2.2.1. Modal Strain Energy. Taking the Euler-Bernoulli hinged slab as an example, the strain energy is

$$
\mathrm{SE}=\left(\frac{1}{2}\right) \int_{0}^{L} \operatorname{EI}(x)\left(\frac{\partial^{2} w(x)}{\partial x^{2}}\right)^{2} \mathrm{~d} x,
$$

where $\mathrm{EI}(x)$ and $L$ are the bending rigidity and length of the hinged slab, respectively. From (4), the modal strain energy of the $j$ th modal vibration mode $\phi_{j}\left(x_{i}\right)$ of a linear hinged slab with $N$ units and $m$ degrees of freedom is

$$
\operatorname{MSE}_{j}=\left(\frac{1}{2}\right) \int_{0}^{L} \operatorname{EI}(x)\left(\frac{\partial^{2} \phi_{j}(x)}{\partial x^{2}}\right)^{2} \mathrm{~d} x .
$$

Based on finite element method basic principle, the $j$ th mode strain energy of the element $i$ is

$$
\operatorname{MSE}_{i j}=\left(\frac{1}{2}\right)\{\phi\}_{j}^{T} k_{i}\{\phi\}_{j},
$$

where $k_{i}$ is the extended order of the element stiffness matrix $k_{i}^{c}$ in the global coordinate system. 
2.2.2. Relationship between Displacement Mode and Strain Mode. The structural damage will change mechanical properties of the materials. This is reflected by the loss of local structural stiffness, resulting in a change of the MSE in the damaged region [13]. In this study, the hinged slab structure is modeled as a planar Euler-Bernoulli hinged slab with a constant cross section. For the $i$ th hinged slab element, the elemental nodal displacement vector $\{d\}^{e}$ has the following form [27]:

$$
\{d\}^{e}=\left\{\begin{array}{llll}
v_{i} & \theta_{i} & v_{i+1} & \theta_{i+1}
\end{array}\right\}^{T},
$$

where the superscripts $e$ and $T$ stand for elemental and transpose, respectively; $v_{i}$ and $v_{i+1}$ are the nodal deflection of the $i$ th and $(i+1)$ th nodes, respectively; and $\theta_{i}$ and $\theta_{i+1}$ are the nodal rotation of the $i$ th and $(i+1)$ th nodes, respectively.

The deflection $w\left(x_{i}\right)$ at local coordinate $x_{i}$ on the hinged slab element can be expressed as

$$
w\left(x_{i}\right)=\{q\}\{d\}^{e},
$$

where $\{q\}$ is the shape function vector.

The strain $\varepsilon\left(x_{i}\right)$ of the local coordinate $x_{i}$ on each hinged slab element can be expressed as

$$
\varepsilon\left(x_{i}\right)= \pm h\left(\frac{\mathrm{d}^{2} w\left(x_{i}\right)}{\mathrm{d} x^{2}}\right)= \pm h\left(\frac{\mathrm{d}^{2}\{q\}}{\mathrm{d} x^{2}}\right)\{d\}^{e},
$$

where $h$ represents the distance from the neutral axis of the hinged slab to the location of the strain gauge.

To develop a damage indicator, the displacement mode and rotation mode are replaced by the strain mode, and the nodal displacement vector of the $i$ th hinged slab element is replaced by a nodal strain vector, including the strains on the structural surface of the $i$ th hinged slab element, i.e., $\varepsilon_{i}$ and $\varepsilon_{i+1}$. Therefore, the nodal strain vector $\{\varepsilon\}$ is

$$
\{\varepsilon\}=\left\{\begin{array}{ll}
\varepsilon_{i} & \varepsilon_{i+1}
\end{array}\right\}^{T} .
$$

The relationship between the nodal displacement vector $\{d\}^{e}$ and the nodal strain vector $\{\varepsilon\}$ can be derived as follows:

$$
\{d\}^{e}=[T]\{\varepsilon\}=[T]\left\{\varepsilon_{i} \varepsilon_{i+1}\right\}^{T},
$$

where $[T]$ is the elemental transfer matrix:

$$
[T]=\frac{1}{3 h\left(l^{2}+4\right)}\left[\begin{array}{cc}
l^{2} & -l^{2} \\
6 l+2 l^{2} & 6 l+l^{3} \\
-l^{2} & l^{2} \\
-6 l-l^{3} & -6 l-2 l^{3}
\end{array}\right] .
$$

In modal strain analysis, the strain mode and displacement mode are coupled with the same transfer matrix $[T]$. Therefore, the relationship between the $j$ th displacement mode shape on the $i$ th element $\left\{\phi_{i j}\right\}$ and the $j$ th strain mode shape on the $i$ th element $\left\{\phi_{i j}^{\varepsilon}\right\}$ can be expressed as follows [28]:

$$
\left\{\phi_{i j}\right\}=[T]\left\{\phi_{i j}^{\varepsilon}\right\}
$$

2.2.3. Elemental MSE of Strain Mode. Substituting (10) into (12), the elemental MSE of the strain mode can be calculated as follows:

$$
\begin{aligned}
\operatorname{MSE}_{i j} & =\frac{1}{2}\left\{\phi_{i j}^{\varepsilon}\right\}[T]^{T} k_{i}[T]\left\{\phi_{i j}^{\varepsilon}\right\}, \\
& =\frac{1}{2}\left\{\phi_{i j}^{\varepsilon}\right\}\left[K_{i}\right]\left\{\phi_{i j}^{\varepsilon}\right\},
\end{aligned}
$$

where $\left[K_{i}\right]$ is defined as

$$
\left[K_{i}\right]=[T]^{T} k_{i}[T]=\left(\frac{2 l \mathrm{EI}}{3 h^{2}}\right)\left[\begin{array}{ll}
2 & 1 \\
1 & 2
\end{array}\right] .
$$

From (11) through (16), the elemental MSE before and after damage can be represented by the strain mode as

$$
\begin{aligned}
\operatorname{MSE}_{u i j} & =\sum_{j=1}^{m}\left\{\phi_{u i j}^{\varepsilon}\right\}^{T}\left[K_{i}\right]\left\{\phi_{u i j}^{\varepsilon}\right\} . \\
\mathrm{MSE}_{d i j} & =\sum_{j=1}^{m}\left\{\phi_{d i j}^{\varepsilon}\right\}^{T}\left[K_{i}\right]\left\{\phi_{d i j}^{\varepsilon}\right\},
\end{aligned}
$$

where $\phi_{u i j}^{\varepsilon}$ and $\phi_{d i j}^{\varepsilon}$ are $j$ th strain mode shape on the $i$ th element before and after damage, respectively. Because the elemental stiffness of damaged elements is unknown, the undamaged elemental stiffness matrix $\left[K_{i}\right]$ is used as an approximation instead of the damaged one in $\mathrm{MSE}_{d i j}$.

\section{Structural Damage Identification Index}

3.1. Identification of Hinge Joint Damage. According to the basic theory of local information entropy [29, 30], when calculating the local probability of a hinge joint element $i$, the strain energy of the adjacent elements around $i$ should be considered. Considering the strain energy of each element, $W$, as the information amount, the local probability $P_{i}^{j}$ of element $i$ at the $j$ th hinge joint can be expressed as follows:

$$
P_{i}^{j}=\left(\frac{W_{i}^{j}}{\sum_{m=j-i}^{j} \sum_{n=i-1}^{i} W_{s n}^{m}+\sum_{n=i-1}^{i+1} W_{n}^{j}}\right),
$$

where $W_{i}^{j}$ and $W_{n}^{j}$ are the deformation energies of the $i$ and $n$ elements at the $j$ th hinge joint, respectively, and $W_{s n}^{m}$ is the strain energy of element $n$ on the $m^{\text {th }}$ concrete hinged slab.

For the $j$ th hinge joint, a new variable $\lambda_{i}^{j}$ is introduced to stand for the weight of the $i$ element local probability.

$$
\lambda_{i}^{j}=\left(\frac{P_{i}^{j}}{\sum_{k=1}^{n} P_{k}^{j}}\right)\left(\sum_{k=1}^{n} \lambda_{k}^{j}=1\right) .
$$

During the calculation, the larger $n$ is, the more data near support elements will be discarded. By substituting (19) into (2), the local energy information entropy $H_{i}^{j}$ of element $i$ of the $j$ th hinge joint can be expressed as 


$$
H_{i}^{j}=\frac{p_{i}^{j}}{\sum_{k=1}^{n} P_{k}^{j}}\left(\ln \sum_{k=1}^{n} P_{k}^{j}-\ln P_{i}^{j}\right) .
$$

Subsequently, the damage degree $D_{i}^{j}$ of the $i$ element at the $j$ th hinge joint is calculated as

$$
D_{i}^{j}=\left\{\begin{array}{cc}
0 & H_{i, u}^{j} \leq H_{i, d}^{j} \\
1-\frac{H_{i, d}^{j}}{H_{i, u}^{j}} H_{i, u}^{j}>H_{i, d}^{j}
\end{array}\right\},
$$

where $H_{i, u}^{j}$ and $H_{i, d}^{j}$ are the local energy information entropy of element $i$ of the $j$ th hinge joint before and after damage, respectively.

3.2. Identification of Hinged Slab Damage. Based on (16) and (17), the modal strain ratio energies before and after the damage can be, respectively, defined as new physical quantities $\lambda_{u i}$ and $\lambda_{d i}$ :

$$
\begin{aligned}
& \lambda_{u i}=\frac{\operatorname{MSE}_{u i j}}{\sum_{i=1}^{n} \mathrm{MSE}_{u i j}} . \\
& \lambda_{u i}=\frac{\mathrm{MSE}_{u i j}}{\sum_{i=1}^{n} \mathrm{MSE}_{u i j}} .
\end{aligned}
$$

Substituting (22) and (23) into (2), the modal strain ratio energy entropy functions $S_{u i}$ and $S_{d i}$ of element $i$ before and after damage are, respectively, established as follows:

$$
\begin{aligned}
S_{u i} & =-\sum_{i=1}^{n} \lambda_{u i} \ln \lambda_{u i}, \\
& =-\sum_{i=1}^{n} \frac{\mathrm{MSE}_{u i j}}{\sum_{i=1}^{n} \mathrm{MSE}_{u i j}} \ln \frac{\mathrm{MSE}_{u i j}}{\sum_{i=1}^{n} \mathrm{MSE}_{u i j}}, \\
S_{d i} & =-\sum_{i=1}^{n} \lambda_{d i} \ln \lambda_{d i}, \\
& =-\sum_{i=1}^{n} \frac{\mathrm{MSE}_{d i j}}{\sum_{i=1}^{n} \mathrm{MSE}_{d i j}} \ln \frac{\mathrm{MSE}_{d i j}}{\sum_{i=1}^{n} \mathrm{MSE}_{d i j}} .
\end{aligned}
$$

The entropy difference can be calculated as follows:

$$
S_{i}=S_{d i}-S_{u i}
$$

At the site of damage, the value of $S_{i}$ will change significantly compared to a site with no damage. Therefore, we can easily identify the location and severity of damage elements.

\section{Finite Element Numerical Simulation}

Due to the existence of hinge joints, the force transfer mechanism of a simply supported beam is different from that of other types of bridges. The hinge joint is usually in a complicated stress state with bending moment $m(x)$, shear $g(x)$, and compression $c(x)$. The hinge joint is a crucial component to ensure that the slabs work together, as shown in Figure 1.

Local strain energy information entropy and modal strain energy information entropy are used in this paper to identify the main damage in hinge joints and hinged slabs, respectively.

4.1. Mechanical Model of Hinge Joint. It is obvious that traditional hinge plate theory cannot define the actual force at the hinge joint because it only considers shear force. The force diagram in Figure 1 can be simplified, as shown in Figure 2. Since $m(x)$ and $c(x)$ mainly act as transverse normal stresses on the hinge joints, they are equivalent to a pair of equal and opposite transverse forces $n a(x)$ and $n b(x)$.

Bearing shear action is the main function of hinge joints. However, due to the small size of the hinge joints, it is assumed that the shear force is evenly distributed at the hinge joints. Moreover, when the hinge joints are compressed due to the deformation of the concrete plates, the directions of the upper and lower parts of the hinge joints may be inconsistent; there are two kinds of forces: tension and compression. Therefore, the hinge joint is equivalent to two springs $a$ and $b$, which only bear transverse tension or pressure, and a connecting rod system which only bears vertical shear, as shown in Figure 3.

For the entire beam, the calculation model of the hinge joints and hinged slabs can be obtained by segmenting and simulating the hinge joints of each section, as shown in Figure 3, with numbered elements, as shown in Figure 4.

4.2. Finite Element Analysis Model Establishment. The finite element analysis model was established in ANSYS, which consists of three pieces of concrete hinged slabs connected with the hinge joints 1 and 2 . The width and height of each hinged slab are $950 \mathrm{~mm}$ and $800 \mathrm{~mm}$, respectively.

The span is $10 \mathrm{~m}$ and is divided into 50 elements longitudinally. The width $c$ and height $h$ of the hinge joints are $75 \mathrm{~mm}$ and $700 \mathrm{~mm}$, respectively. The concrete grade is C40. The hinge joints are composed of two springs subjected only to transverse forces and one spring that is subjected only to vertical forces. The spring stiffnesses are $k_{a b i}$ and $k_{g i}$, respectively, as shown in Figure 5.

$$
\begin{aligned}
& k_{a b i}=\frac{1}{2 c} E h l^{e} . \\
& k_{g i}=\frac{1}{2 \alpha c} G h .
\end{aligned}
$$

Here, $E$ is the elastic modulus of concrete; $G$ is the shear modulus of concrete; $l^{e}$ is the length of the partition section, $l^{e}=200$; and $\alpha$ is the rectangular section of constant maturity, $\alpha=1.5$.

When the finite element model is loaded, a concentrated force $\mathrm{Pi}=100 \mathrm{kN}$ is applied at the center of the structure. However, to avoid the occurrence of a concentrated strain in the structure, according to the method in literature [31], a 


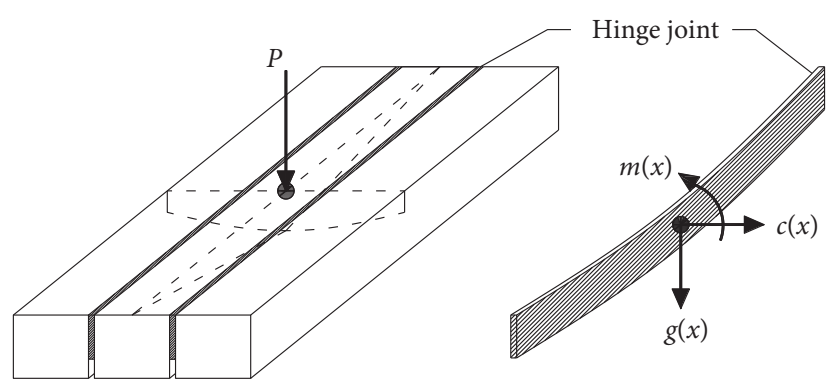

FIGURE 1: Force diagram of simply supported beam.

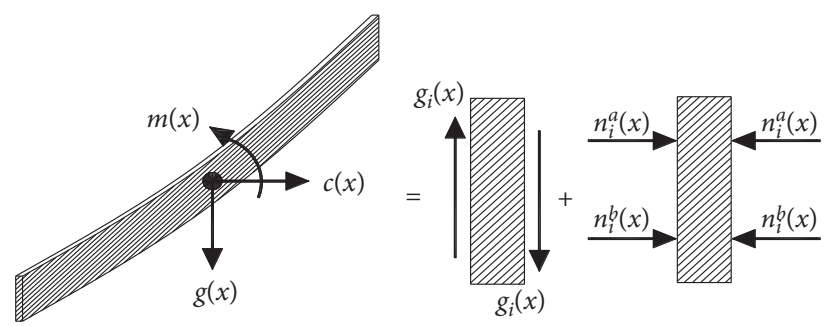

Figure 2: Simplification of the forces at hinge joints.

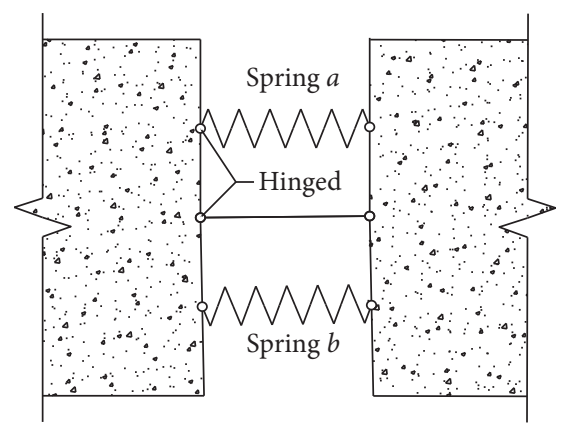

Figure 3: Hinge joint model.

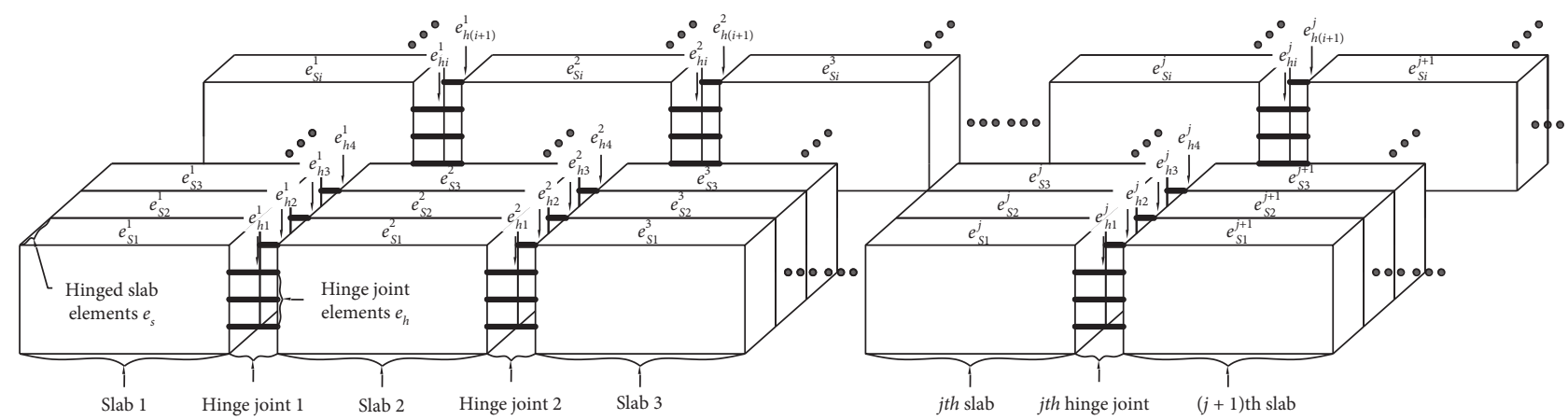

FIGURE 4: Structure segmentation and unit numbering.

sinusoidal uniformly distributed load $p(x)$ of a half-wave was used instead. The loaded structure is shown in Figure 6.

$$
p(x)=\left(\frac{2 p_{i}}{L}\right) \sin \left(\frac{\pi x}{L}\right) .
$$

\section{Results of Damage Identification Analysis}

5.1. Analysis of Hinge Joint Damage. Through the above theory, the strain energy information entropy index value of 


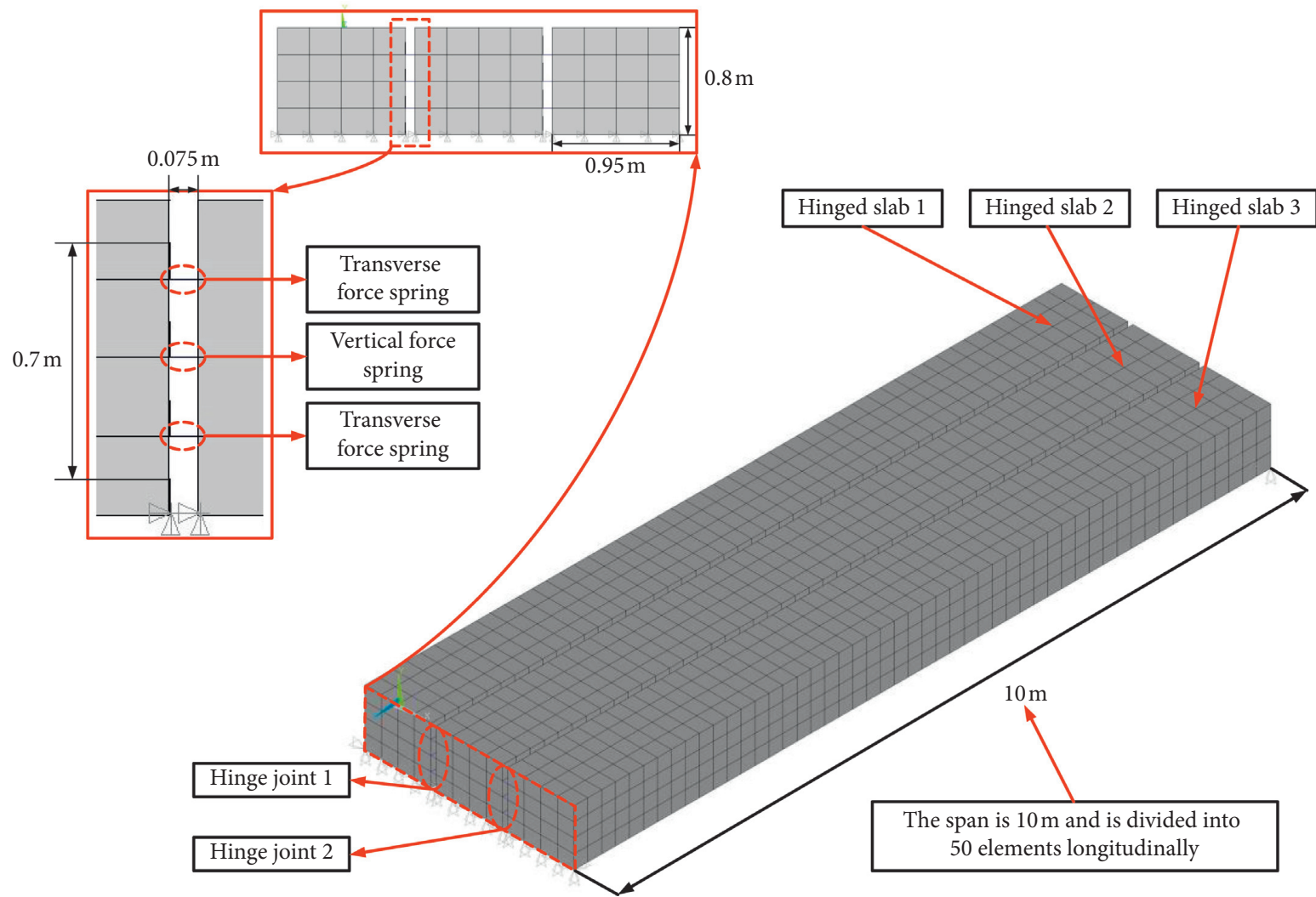

Figure 5: Finite element analysis model.

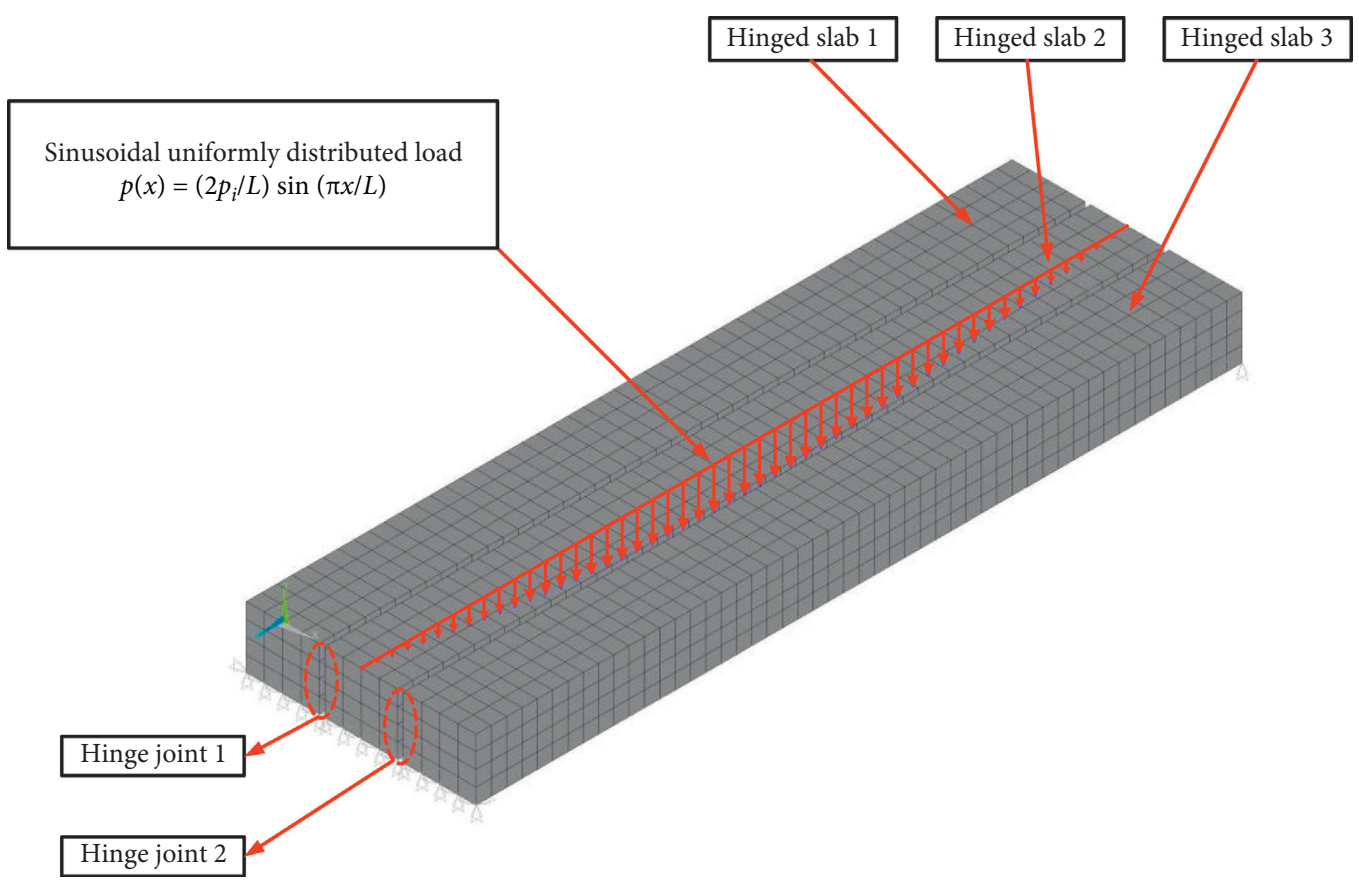

FIgURE 6: Finite element analysis model loading.

each damage case is calculated in MATLAB programming environment.

In this paper, different damage cases were set, as shown in Table 1.
5.1.1. Single-Point Damage of Single-Hinge Joint. In the first case study, the damage is simulated by reducing the stiffness of the joint element, and the damage degree is preset at $5 \%$. Figure 7 shows the analysis results. From the results, the 
TABle 1: Damage cases in hinge joints.

\begin{tabular}{|c|c|c|}
\hline Damage case & Damage element no. & Damage severity (\%) \\
\hline 1 & $e_{h 26}^{1}$ & 5 \\
\hline \multirow{2}{*}{2} & $e_{h 26}^{1}$ & 5 \\
\hline & $e_{h 38}^{1}$ & 10 \\
\hline \multirow{3}{*}{3} & $e_{h 26}^{1}$ & 5 \\
\hline & $e_{h 38}^{1}$ & 10 \\
\hline & $e_{h 39}^{1}$ & 10 \\
\hline \multirow{3}{*}{4} & $e_{h 26}^{1}$ & 10 \\
\hline & $e_{h 38}^{1}$ & 15 \\
\hline & $e_{h 12}^{2}$ & 5 \\
\hline
\end{tabular}

Note. $e_{h 26}^{1}$ represents element 26 of the first hinge joint.

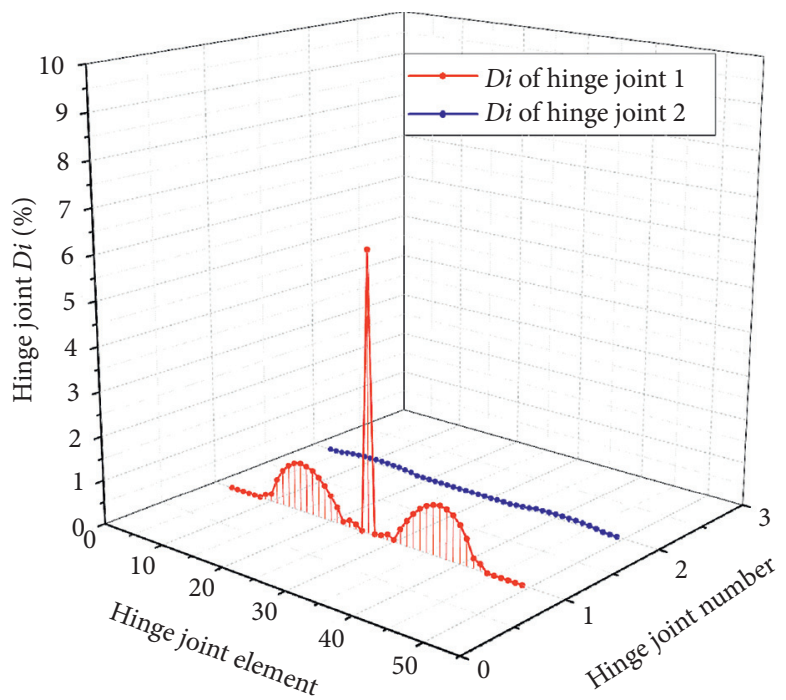

FIGURE 7: Calculation results of damage degree of hinge joints for case study 1.

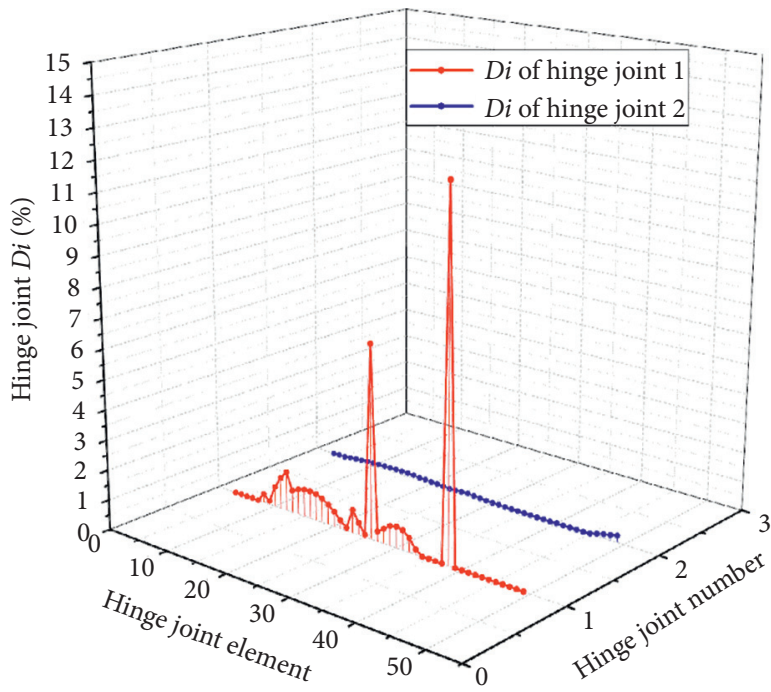

(a)

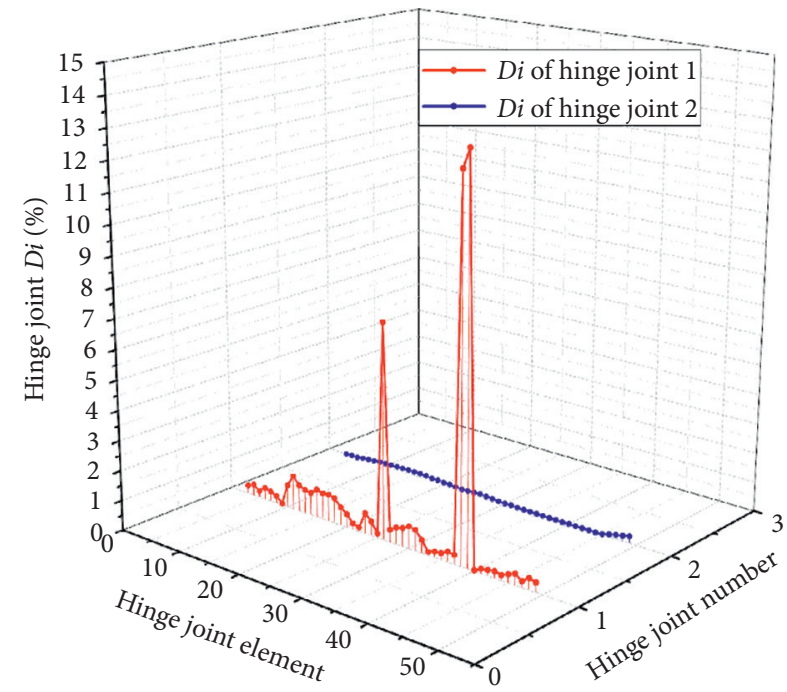

(b)

Figure 8: Calculation results of damage degree of hinge joints. (a) Case study 2. (b) Case study 3. 
damage position of the 26th hinge joint element of hinge joint 1 can be clearly seen, and the damage degree is $6.26 \%$, with an identification error of $20.12 \%$.

The results show that the hinge joint damage identification method developed in this paper can accurately identify the location of single-hinge joint damage, and the calculated damage degree is higher than the preset damage degree.

5.1.2. Multipoint Damage of Hinge Joints. Figures 8 and 9 show the results for the case studies 2,3 , and 4 . The hinge joint damage identification method proposed in this paper is also effective for detecting the multipoint damage of hinge joints. It can be clearly seen from the results that the damage position is the same as the preset damage position. In calculation results of damage degree of case study 3 , continuous damage appears in the 38th and 39th hinge joint elements, which has a significant impact on the damage identification result of the 26th hinge joint element, and the damage identification error increases.

When two hinge joints are damaged at the same time, the result of damage identification is shown in Figure 9. It can be seen that the damage index can accurately locate the damage position of the two hinge joints, but the calculated damage degree error is slightly larger.

Table 2 shows the calculated damage degree values.

5.2. Analysis of Hinged Slab Damage. Hinged slab 1 was removed to identify local damage. Similarly, the elastic modulus of the structure was reduced to simulate damage. In this section, the first three modes of the structure are evaluated.

The noise interference is inevitable in actual engineering, so it is necessary to verify the antinoise performance of the proposed method. Theoretically, it is often assumed that the variation of errors follows a Gaussian distribution, but the Gaussian distribution contradicts the boundedness of actual errors. Therefore, the truncated Gaussian distribution (TGD) error model is introduced [32], and the model of random variables subject to the Gaussian distribution is truncated. The simulation error is introduced as follows:

$$
\Phi_{i}^{e}=\Phi_{i}[1+u \cdot \operatorname{TGD}(-1,1)]
$$

where $\Phi_{i}$ and $\Phi_{i}^{e}$ represent the mode shape components with and without noise error, respectively; $u$ is the noise level. $\operatorname{TGD}(-1,1)$ are random variables subject to TGD; the truncated upper and lower limits are taken as -1 and 1 , respectively. Table 3 defines each damage case.

For case studies 5 and 6, element 25 is assumed to have damage, represented by reductions in the elasticity modulus equal to $10 \%$ and $30 \%$, respectively.

The results of damage detection shown in Figure 10 indicate that singular damage can be identified from the strain energy data with the proposed method successfully. When $10 \%$ and $30 \%$ damage occurred to the structure, the damage index values were 0.0075 and 0.029 , respectively. With this linear relationship, the severity of the damage can be evaluated by calibration.

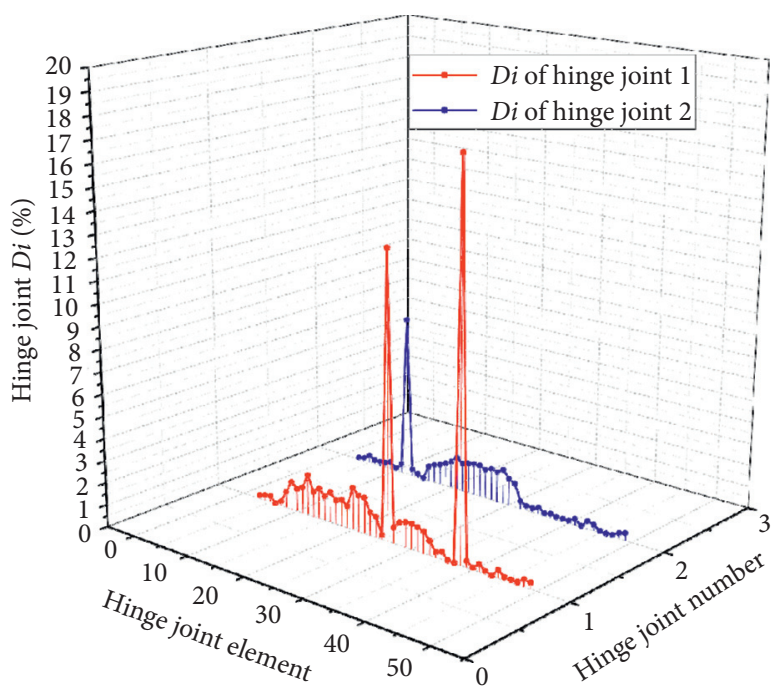

FIgUre 9: Calculation results of damage degree of hinge joints for case study 4.

TABLE 2: Damage degree values for multipoint damage case studies.

\begin{tabular}{lccc}
\hline $\begin{array}{l}\text { Damage } \\
\text { case }\end{array}$ & $\begin{array}{c}\text { Damage } \\
\text { element no. }\end{array}$ & $\begin{array}{c}\text { Damage degree } \\
\text { values (\%) }\end{array}$ & $\begin{array}{c}\text { Identification } \\
\text { error }(\%)\end{array}$ \\
\hline 2 & $e_{h 26}^{1}$ & 6.44 & 22.36 \\
& $e_{h 38}^{1}$ & 12.08 & 17.21 \\
\hline \multirow{3}{*}{3} & $e_{h 26}^{1}$ & 7.14 & 29.97 \\
& $e_{h 38}^{1}$ & 12.41 & 19.41 \\
& $e_{h 39}^{1}$ & 13.06 & 23.43 \\
\hline \multirow{3}{*}{4} & $e_{h 26}^{1}$ & 12.88 & 25.79 \\
& $e_{h 38}^{1}$ & 17.40 & 19.45 \\
& $e_{h 12}^{2}$ & 7.42 & 24.56 \\
\hline
\end{tabular}

TABle 3: Damage case studies in the hinged slab.

\begin{tabular}{lcc}
\hline Damage case & Damage element no. & Damage severity (\%) \\
\hline 5 & 25 & 10 \\
\hline 6 & 25 & 30 \\
\hline 7 & 10,35 & 10 \\
\hline 8 & 10,35 & 30 \\
\hline 9 & 10,35 & $30(5 \%$ noise $)$ \\
\hline 10 & $5,15,25,35$ & $30(10 \%$ noise $)$ \\
\hline 11 & $5,15,25,35$ & $20,20,20,20$ \\
\hline 13 & $5,15,25,35$ & $10,20,30,40$ \\
\hline
\end{tabular}

Note. "10, 30 (5\% noise)" represents an addition of 5\% noise error in the damage case study.

Case studies 7 and 8 were used to study double-damage cases. For case studies 7 and 8, elements 10 and 35 are assumed to have a stiffness reduction equal to $10 \%, 10 \%$, and $10 \%, 30 \%$, respectively. Meanwhile, the noise of $5 \%$ and $10 \%$ was introduced into for case study 7 to verify the antinoise performance of the damage index.

The results of damage detection are shown in Figures 11 and 12. The damage location can be accurately identified, and the severity of damage can be evaluated with the linear 


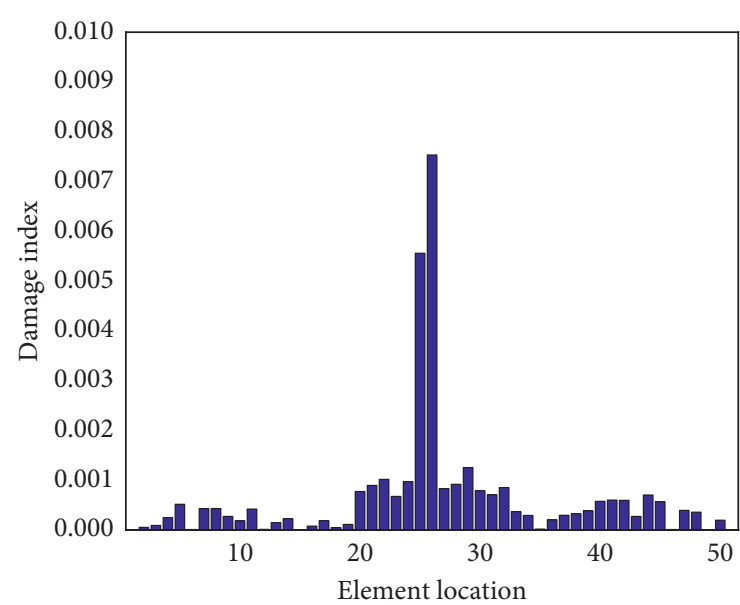

(a)

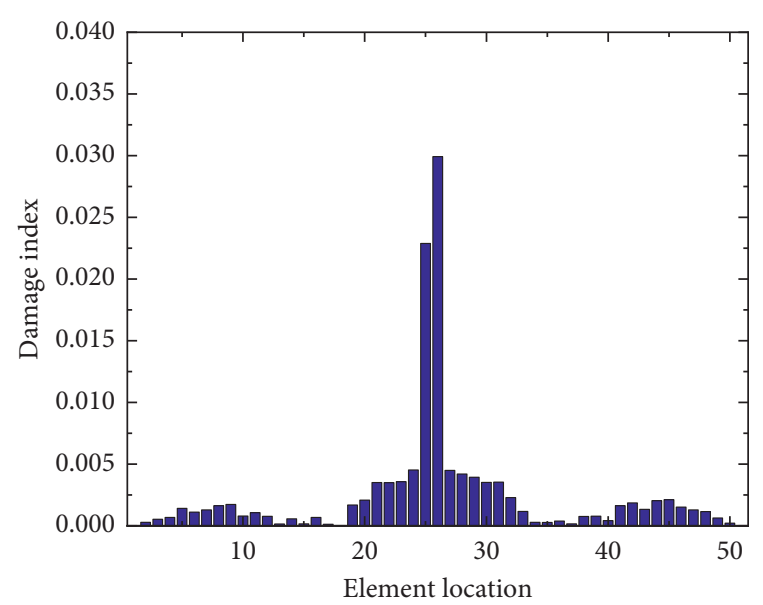

(b)

Figure 10: Damage detection results of single damage cases. (a) Case study 5. (b) Case study 6.

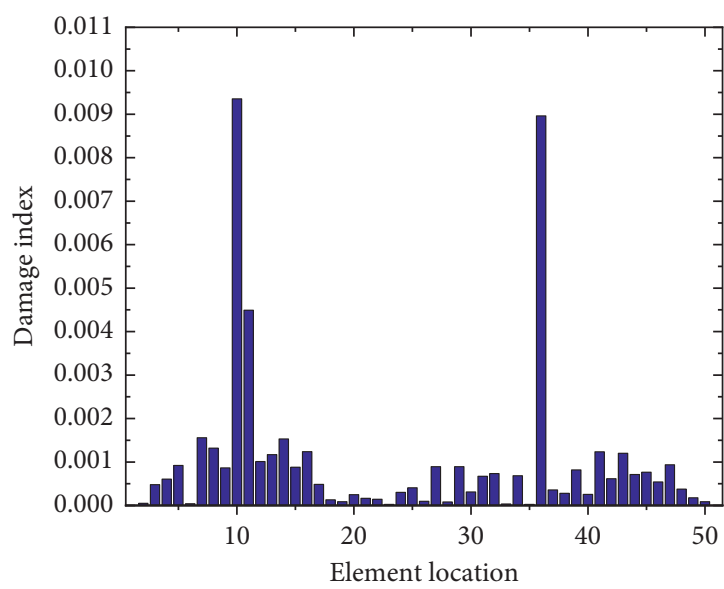

(a)

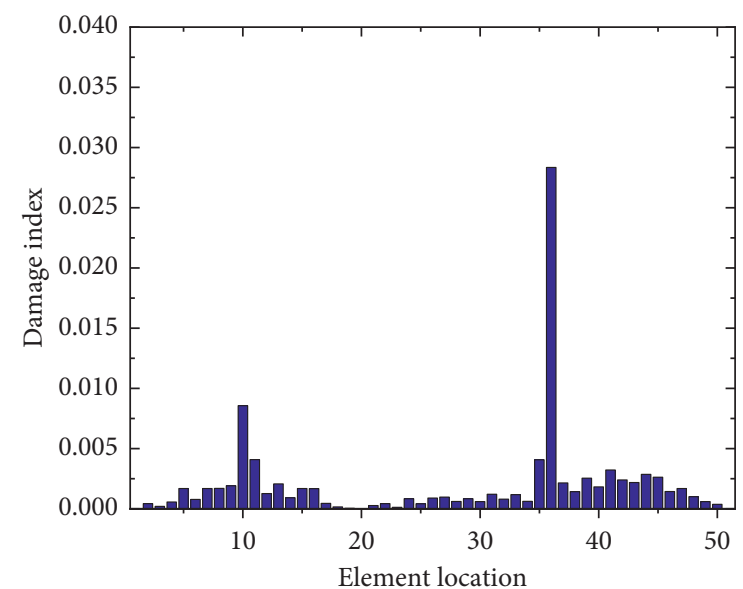

(b)

Figure 11: Damage detection results of double-damage cases. (a) Case study 7. (b) Case study 8.

relationship between the values of the peak index and extent of the damage.

Figure 11 shows that, for case study $7,10 \%$ damage occurred in elements 10 and 35 with damage indexes of 0.0095 and 0.0093 , respectively. Note that the difference is small. For case study $8,10 \%$ and $30 \%$ damage occurred in elements 10 and 35 , respectively. The corresponding damage indexes are 0.0086 and 0.028 , and the linear relationship of the damage index approaches the true extent of the damage.

Figure 12 shows that the proposed damage index can still locate the damage position and judge the damage degree well under noise intensities of $5 \%$ and $10 \%$.

In order to verify the effectiveness of the index in more complex damage cases, different damage cases are set in elements $5,15,25$, and 35 of the hinged slab, respectively; the damage identification results are shown in Figure 13. For case study 11 , the damage degree of the damage position was all $20 \%$, and the damage index values were $0.0201,0.0192,0.0213$, and 0.0189 , respectively. For case study 12 , the damage degree of elements increased from $10 \%$ to $40 \%$ in turn, and the

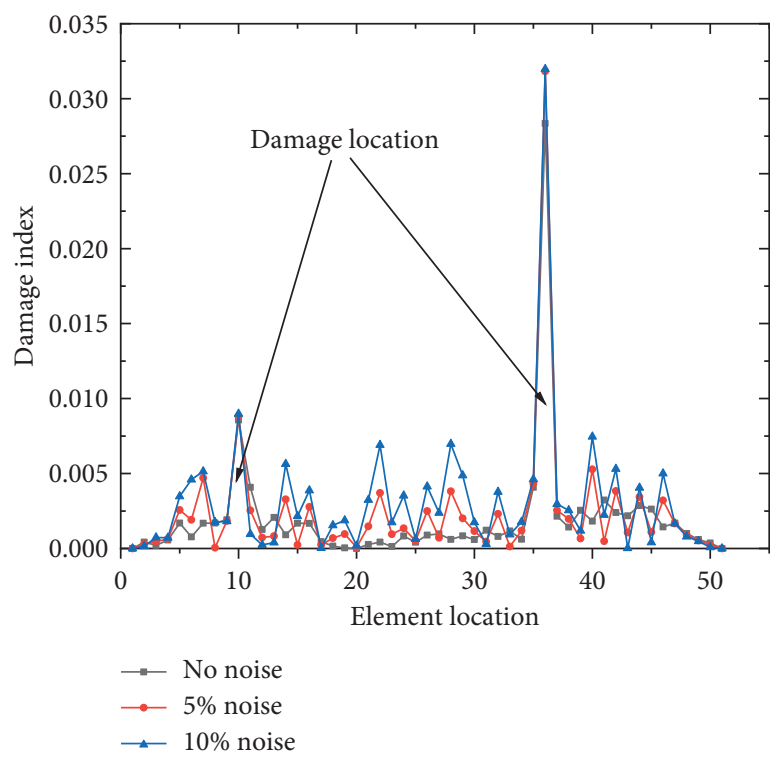

Figure 12: Antinoise verification of damage index. 


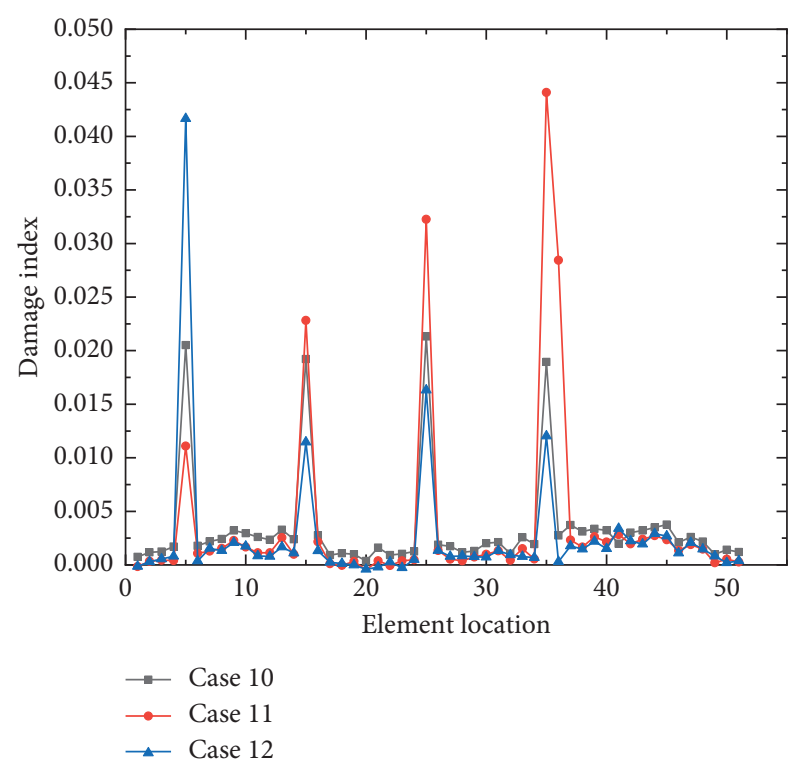

FIgURE 13: Damage detection results of cases 10-12.

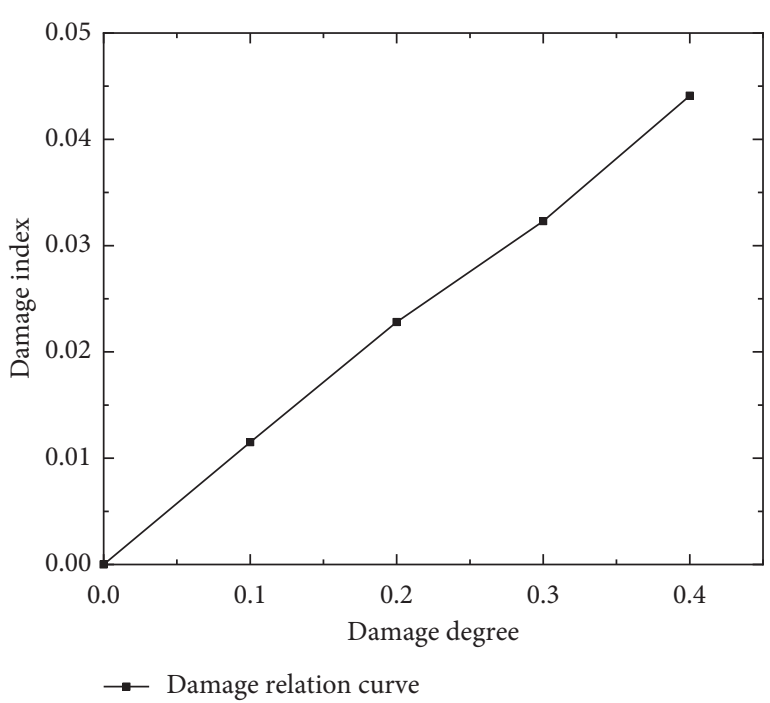

Figure 14: Damage relation curve of case 11.

corresponding index values were $0.011,0.023,0.032$, and 0.044 , which basically showed a linear increase; the damage relationship curve is shown in Figure 14. It can be seen that the damage index is sensitive to all damage locations and can well reflect the damage degree of the structure.

\section{Conclusions}

In this paper, the strain energy entropy index was proposed by combining the strain energy and information entropy theories. Based on the new index used in damage identification of a simply supported beam, the following conclusions can be drawn.

According to the finite element analysis and calculation, the proposed method can locate well single-point and multipoint damage of hinge joints. Moreover, the hinge joint damage identification method is less affected by other hinge joints with this method, showing strong stability. When calculating the extent of hinge joint damage, the calculated value is often higher than the set value. This identification error is the next topic to study in future works.

The modal strain energy can accurately identify the damage location of the hinged slab and effectively judge the extent of the damage by the peak value of the index, and it is effective despite the noise. However, the stress form of the simply supported beam is relatively complex. Therefore, the practicability of the new index for this kind of structure should be further improved.

The stress analysis and hinge joint simulation of a simply supported beam should be further explored. For accurate calculation of the extent of hinge joint damage, artificial intelligence methods are promising choices, such as artificial neural network (ANN). The damage identification method based on artificial intelligence well be the focus of future works.

\section{Data Availability}

All data are available within the article or can be obtained from the corresponding author upon request.

\section{Conflicts of Interest}

The authors declare that there are no conflicts of interest regarding the publication of this paper.

\section{Acknowledgments}

This work was supported by the Key Natural Science Project of Anhui Provincial Education Department (Grant no. KJ2019A0746), Natural Science Foundation of China (Grant no. 51868045), Natural Science Foundation Youth Fund of Anhui Province (Grant no. 2008085QE247), and Startup Fund for Doctor of Anhui Jianzhu University (Grant no. 2019QDZ08).

\section{References}

[1] T. Yamane, M. K. Tadros, and P. Arumugasamy, "Short to medium span precast prestressed concrete bridges in Japan," PCI Journal, vol. 39, no. 2, pp. 74-100, 1994.

[2] H. H. Hussein, S. M. Sargand, I. Khoury et al., "Environmentinduced behavior of transverse tie bars in adjacent prestressed box-girder bridges with partial depth shear keys," Journal of Performance of Constructed Facilities, vol. 31, no. 5, pp. 04017074-1-13, 2017.

[3] H. H. HusseinS. M. Sargand et al., "Experimental validation of optimized ultrahigh performance concrete shear key shape for precast pre-stressed adjacent box girder bridges," Construction and Building Material, vol. 90, pp. 178-190, 2018.

[4] S. Alampalli and E. F. Dicocco, "Performance of full-depth shear keys in adjacent prestressed box beam bridges," $P C I$ Journal, vol. 43, no. 2, pp. 72-79, 1998.

[5] Q. Wang, Q.-x. Wu, and C. Bao-chun, "Experimental study on failure mode of hinged joint in assembly voided slab bridge," Engineering Mechanics, vol. 31, no. 3, pp. 115-120, 2014. 
[6] Q.-x. Wu, C. Yue-chi, and C. Kang-ming, "Failure mode analysis of hinged voided slab with gate-type steel rebars at bottom of junction sur-face," Journal of Traffic and Transportation Engineering, vol. 15, no. 5, pp. 15-25, 2015.

[7] Q. jin-tao and H. ming-fei, "Disease statistics and analysis of expressway hollow slab bridges," Highway Traffic Technology, vol. 34, no. 1, pp. 88-91, 2018.

[8] O. S. Salawu, "Detection of structural damage through changes in frequency: a review," Engineering Structures, vol. 19, no. 9, pp. 718-723, 1997.

[9] A. Pandey, M. Biswas, and M. Samman, "Damage detection in curvature mode shapes," Journal of Sound and Vibration, vol. 145, no. 2, pp. 321-332, 1991.

[10] H. Zhu, L. Li, and X. Q. He, "Damage detection method for shear buildings using the change in first mode shape slopes," Computers \& Structures, vol. 89, no. 9, pp. 733-743, 2011.

[11] K. V. Nguyen, "Mode shapes analysis of a cracked beam and its application for crack detection," Journal of Sound and Vibration, vol. 333, no. 3, pp. 848-872, 2014.

[12] K. Y. Koo, S. H. Sung, and H. J. Jung, "Damage quantification of shear buildings using deflections obtained by modal flexibility," Smart Materials and Structures, vol. 20, no. 4, Article ID 045010, 2011.

[13] Z. Y. Shi, S. S. Law, and L. M. Zhang, "Structural damage localization from modal strain energy change," Journal of Sound and Vibration, vol. 218, no. 5, pp. 825-844, 1998.

[14] Z. Y. Shi, S. S. Law, and L. M. Zhang, "Improved damage quantification from elemental modal strain energy change," Journal of Engineering Mechanics, vol. 128, no. 5, pp. 521-529, 2002.

[15] S. M. A. Seyedpoor, "Two stage method for structural damage detection using a modal strain energy based index and particle swarm optimization," International Journal of Non-linear Mechanics, vol. 47, no. 1, pp. 1-8, 2012.

[16] M. Jahangiri, M. A. Najafgholipour, S. M. Dehghan et al., "The efficiency of a novel identification method for structural damage assessment using the first vibration mode data," Journal of Sound and Vibration, vol. 458, no. 1, pp. 1-16, 2019.

[17] Wu Shao-qing, J.-x. Zhou, R. Shen et al., "Reformulation of elemental modal strain energy method based on strain modes for structural damage detection," Advances in Structural Engineering, vol. 20, no. 6, pp. 1-10, 2016.

[18] C. Pei-jang, C.-s. Gu, and J. Wang, "Application of entropy theory in the analysis of concrete crack propagation process," Journal of Basic Science and Engineering, vol. 16, no. 1, pp. 50-56, 2008.

[19] C.-h. Xu and Q.-w. Ren, "Study on entropy mutation criterion for stability analysis of sur-rounding rock," Rock and Soil Mechanics, vol. 25, no. 3, pp. 437-440, 2004.

[20] J.-d. Zheng, M.-j. Chen, J.-s. Cheng et al., "Multiscale fuzzy entropy and its application in rolling bearing fault diagnosis," Journal of Vibration Engineering, vol. 27, no. 1, pp. 145-151, 2014.

[21] L. Guo-hua and Z.-g. Wu, "New thought on dynamic identification technology for damage detection of RC structures by introducing information entropy theory," Journal of Vibration and Shock, vol. 30, no. 6, pp. 162-171, 2011.

[22] Z.-k. Xie and L. Guo-hua, "Application of ap-proximate entropy in concrete strutures damage identification," Journal of Zhejiang University, vol. 47, no. 3, pp. 456-463, 2013.

[23] C. Pei-jiang, C.-s. Gu, and Y.-c. Gu, "Maximum entropy method for dam safety monitoring index," Geomatics and Information Science of Wuhan University, vol. 33, no. 11, pp. 1126-1129, 2008.
[24] X. Chang-sheng, M. Fu-chao, Y. Zhou et al., "Application of generalized local information entropy in damage identification of simply supported beams," Journal of Gansu Science, vol. 29, no. 6, pp. 89-94, 2017.

[25] J. Chen, Y. Cao, and B. Duan, "Information Entropy of structure and maximum entropy principle," Journal of Applied Mechanic, vol. 29, no. 4, pp. 116-121, 1998.

[26] D. Jiang, Information Theory and Coding, University of Science and Technology of China Press, Hefei, China, 2009.

[27] $\mathrm{Z}$. $\mathrm{Xu}$ and $\mathrm{K}$. Wu, "Damage detection for space truss structures based on strain mode under ambient excitation," Journal of Engineering Mechanics, vol. 138, no. 10, pp. 1215-1223, 2012.

[28] L. H. Yam and J. C. Golinval, "Theoretical and experimental study of modal strain analysis," Journal of Sound and Vibration, vol. 191, no. 2, pp. 251-260, 1996.

[29] X. Chang-sheng, J.-s. Cheng, and Yu Zhou, "Study on the entropy change of concrete crack evolution based on dissipative system," Journal of Lanzhou University of Technology, vol. 43, no. 3, pp. 122-126, 2017.

[30] Z. Yang, X. Chen, Y. Jiang et al., "Generalized local entropy analysis for crack detection in beam-like structures," Nondestructive Testing and Evaluation, vol. 29, no. 2, pp. 133-153, 2014.

[31] G. H. Li and D. Shi, Calculation of Transverse Load Distribution of Highway and Bridge, People's Communications Press, Beijing, China, 1987.

[32] Y. Zhang, D. Wang, Z. L. Sun et al., "Stress-life model of truncated distribution," Journal of Northeastern University, vol. 21, no. 2, pp. 165-168, 2000. 